\title{
ENERGY DECAY \\ FOR HYPERBOLIC THERMOELASTIC SYSTEMS OF MEMORY TYPE
}

\author{
$\mathrm{BY}$ \\ LUCI HARUE FATORI (Department of Mathematics, Universidade Estadual de Londrina, \\ 86.051-990 Londrina-PR, Brazil) \\ AND
}

JAIME E. MUÑOZ RIVERA (National Laboratory for Scientific Computation, Dept. of Applied and Computational Mathematics, Rua Getulio Vargas 333, Quitandinha 25651-070, Petrópolis RJ, Brasil, and IM, Federal University of Rio de Janeiro)

Abstract. In this paper we study the hyperbolic thermoelastic system, which is obtained when, instead of Fourier's law for the heat flux relation, we follow the linearized model proposed by Gurtin and Pipkin concerning the memory theory of heat conduction. In this case the thermoelastic model is fully hyperbolic. We show that the linear system is well posed and that the solution decays exponentially to zero as time goes to infinity.

1. Introduction. In this work we study the asymptotic behaviour as time goes to infinity of solutions of the thermoelastic hyperbolic system. In the classical linear theory of thermoelasticity, Fourier's law is used to describe the heat conduction in the body. This theory has two principal shortcomings. First, it is unable to account for memory effect which may prevail in some materials, particularly at low temperatures. Secondly, the corresponding parabolic part of the system predicts an unrealistic result, that a thermal disturbance at one point of the body is instantly felt everywhere in the body. These observations lead one to believe that for materials with memory, Fourier's law is not a good model and we have to look for another more general constitutive assumption relating the heat flux to the material thermal history.

The model we study here is related to the following linearized constitutive equations:

$$
\begin{aligned}
\sigma(x, t) & =a \varepsilon(x, t)-\int_{0}^{\infty} g(s) \varepsilon(x, t-s) d s-c \alpha \theta(x, t), \\
q(x, t) & =-K_{0} \theta_{x}(x, t)-\int_{0}^{\infty} k(s) \theta_{x}(x, t-s) d s, \\
e(x, t) & =c_{u} \theta(x, t)+\alpha u_{x}(x, t),
\end{aligned}
$$

Received October 15, 1998.

2000 Mathematics Subject Classification. Primary 74A15, 35L20, 35B40.

Partially supported by CNPq-Brasil. 
where $\sigma$ is the axial stress, $\varepsilon=u_{x}$ is the deformation, $q$ is the heat flux, $e$ is the internal energy, and $\theta$ is the temperature difference from the reference value. The memory kernels $g$ and $k$ are assumed to be regular functions decaying to zero as time goes to infinity. So, the corresponding motion and balance energy equations are given by

$$
\begin{aligned}
\rho_{0} u_{t t}-\operatorname{div} \sigma & =0, \\
e_{t}+\operatorname{div} q & =0,
\end{aligned}
$$

and assuming $\rho_{0}=c_{u}=c=1$, we obtain the following system:

$$
\begin{array}{r}
u_{t t}-a u_{x x}+\int_{-\infty}^{t} g(t-\tau) u_{x x}(\cdot, \tau) d \tau+\alpha \theta_{x}=0, \\
\theta_{t}+K_{0} \theta_{x x}-\int_{-\infty}^{t} k(t-\tau) \theta_{x x}(\cdot, \tau) d \tau+\alpha u_{x t}=0 .
\end{array}
$$

When $K_{0}=0$ and $k>0$ the heat flux relation was proposed by Gurtin and Pipkin [6]. In this case the heat conduction is independent of the present values of the temperature gradient. When $K_{0}>0$ and $k>0$ the heat flux relation was proposed by Coleman and Gurtin [1]: in this model the heat conduction depends on the present values of the temperature gradient. Note that when $k(t)=0$ and $K_{0}>0$ the heat flux relation reduces to Fourier's law.

Let us describe briefly some related results. Concerning the viscoelastic system (without temperature) we have the work of Dafermos [2]. He proved that the solution of the viscoelastic system goes to zero as time goes to infinity without giving explicit rates of decay. Lagnese in [7] considered the linear viscoelastic plate equation obtaining uniform rates of decay for the solution but introducing additional damping terms acting on the boundary. Uniform rates of decay for isotropic viscoelastic materials which occupy the whole $\mathbb{R}^{3}$ were obtained by Dassios [3], provided the relaxation kernel is an exponential function. Dassios's work was improved in $[12,14]$ for bounded and unbounded viscoelastic materials with relaxation function which may decay exponentially or polynomially. For bounded materials the rate of decay of the solution is the same as the rate of decay of the relaxation function, while for materials that occupy the whole space, the rate of decay is like $(1+t)^{-q}$, where $q$ depends on the rate of decay of the relaxation function and the dimension of the space. Concerning the thermoviscoelastic model $(k=0)$ we have the work of Liu and Zheng [8] who proved the exponential stability of the semigroup associated to the system. For the $n$-dimensional case, see Rivera [10]. Finally, when $k(t)>0$ and $K_{0}>0$, Giorgi and Naso [5] proved also the exponential decay of the solution. Here we will study the following model:

$$
\begin{aligned}
u_{t t}-a u_{x x}+\alpha \theta_{x} & =0 \quad \text { in }(0, L) \times \mathbb{R}^{+}, \\
\theta_{t}-k * \theta_{x x}+\alpha u_{x t} & =0 \quad \text { in }(0, L) \times \mathbb{R}^{+}, \\
u(x, 0)=u_{0}(x), \quad u_{t}(x, 0) & =u_{1}(x), \quad \theta(x, 0)=\theta_{0}(x),
\end{aligned}
$$

with the following boundary condition:

$$
u(0, t)=u(L, t)=k * \theta(0, t)=k * \theta(L, t)=0 \quad \forall t>0 .
$$


Note that the above boundary condition implies the Dirichlet boundary condition for $\theta$ provided $\theta \in H^{1}(0, L)$. For simplicity we consider that the initial past history is fixed and is equal to zero,

$$
\theta(x, t)=0, \quad \forall t<0^{-} .
$$

By $*$ we are denoting the convolution product, that is, $k * g(\cdot, t)=\int_{0}^{t} k(t-\tau) g(\cdot, \tau) d \tau$. The system (1.1)-(1.2) is obtained when we consider the memory effect only due to the temperature following Gurtin-Pipkin's model [6]. In this case the model is fully hyperbolic which, in particular, implies the finite speed of propagation of the thermal disturbances and is physically more realistic. From the mathematical point of view, this system is also more interesting than the usual thermoelastic model following Fourier's law, and more interesting than the thermoviscoelastic model of memory type. This is because the dissipation produced by Gurtin-Pipkin's model is weaker than the dissipation in classical thermoelasticity and also weaker than the dissipation produced by thermoviscoelastic materials, which has a damping term due to the stress memory and another because of the temperature. So, in our model, we remove the dissipation given by the stress memory and we only consider the dissipation due to the memory of the heat flux. So, we may ask under such conditions, whether the dissipation produced by the memory of the heat flux is strong enough to produce exponential decay of the solution.

The main result of this paper is to show that the solution of system (1.1)-(1.2) decays exponentially as time goes to infinity provided the kernel $k$ is positive definite and also decays exponentially to zero.

The main difficulty in showing the exponential decay is that the dissipation does not depend on the present values of the heat flux. The boundary condition also plays an important role. When we apply the multiplicative technique, the Dirichlet boundary conditions for both the displacement and the difference of temperature, introduce pointwise terms, which are not possible to estimate using standard Sobolev's inequalities.

Unfortunately, the method used to achieve uniform rates of decay in the aforementioned works is based on the estimates of the present values of the temperature gradient or second-order estimates of the stress memory. Thus, the methods that have been used for establishing uniform rates of decay to thermoelasticity and viscoelasticity, fail in the case of materials with thermal history and a new asymptotic technique has to be devised.

To overcome the above difficulties we use some boundary inequalities to the wave equation, together with some technical ideas involving positive definite kernels. We also introduce two new multipliers (see (4.9)-(4.10) below) which will help us to get the required estimate.

The remaining part of this paper is organized as follows. In section 2 we justify the hyperbolicity of the thermoelastic system. In section 3 we prove the existence and uniqueness of weak solutions and finally, in section 4 we prove that the solution has an exponential decay.

2. The thermoelastic hyperbolic system. In this section we will show that system (1.1)-(1.2) has a hyperbolic behaviour. To do so, we will assume that $a$ and $k(0)$ are positive constants. Let us differentiate equation (1.2) with respect to the time; therefore, 
we have

$$
k(0) \theta_{x x}+k^{\prime} * \theta_{x x}=\theta_{t t}+\alpha u_{x t t} .
$$

Using Volterra's resolvent kernel we can rewrite the above equation as

$$
\theta_{x x}=\frac{1}{k(0)}\left\{\theta_{t t}+\alpha u_{x t t}\right\}+l *\left\{\theta_{t t}+\alpha u_{x t t}\right\},
$$

where $k(0) l$ is the resolvent kernel of $k / k(0)$ and is given by the solution of

$$
k(0) l(t)+k^{\prime} * l=-\frac{k^{\prime}(t)}{k(0)}
$$

and, performing an integration by parts, we get

$$
\begin{aligned}
\theta_{x x}= & \frac{1}{k(0)}\left\{\theta_{t t}+\alpha u_{x x t}\right\}+l(0)\left\{\theta_{t}+\alpha u_{x t}\right\} \\
& -l(t)\left\{\theta_{t}(\cdot, 0)+\alpha u_{x t}(\cdot, 0)\right\}+l^{\prime} *\left\{\theta_{t}+\alpha u_{x t}\right\} .
\end{aligned}
$$

Thereby we have that

$$
\theta_{t t}-k(0) \theta_{x x}+\alpha u_{x x t}=f
$$

where

$$
f=-l(0)\left\{\theta_{t}+\alpha u_{x t}\right\}+l(t)\left\{\theta_{t}(\cdot, 0)+\alpha u_{x t}(\cdot, 0)\right\}-l^{\prime} *\left\{\theta_{t}+\alpha u_{x t}\right\} .
$$

Differentiating equation (1.1) and denoting by $v=u_{t}$ we get that the couple $(v, \theta)$ satisfies

$$
\begin{aligned}
v_{t t}-a v_{x x}+\alpha \theta_{x t} & =0, \\
\theta_{t t}-k(0) \theta_{x x}+\alpha v_{x t} & =f .
\end{aligned}
$$

Since $f$ has only first-order derivatives in $v$ and $\theta$, we consider $f$ as data. In fact, let us suppose we are given the values $v, v_{x}, v_{t}, \theta, \theta_{t}, \theta_{x}$ on a curve $\gamma$. Thus, for $\gamma$ given parametrically by

$$
x=f(s), \quad t=g(s),
$$

we prescribe in $\gamma$ the Cauchy data,

$$
\begin{array}{lll}
v=h_{1}(s), & v_{x}=\varphi_{1}(s), & v_{t}=\psi_{1}(s), \\
\theta=h_{2}(s), & \theta_{x}=\varphi_{2}(s), & \theta_{t}=\psi_{2}(s) .
\end{array}
$$

Therefore, additionally to system (2.1)-(2.2), the second-order derivative of $v$ and $\theta$ satisfies:

$$
\begin{gathered}
v_{x x} f^{\prime}+v_{x t} g^{\prime}=\varphi_{1}^{\prime}, \\
v_{x t} f^{\prime}+v_{t t} g^{\prime}=\psi_{1}^{\prime}, \\
\theta_{x x} f^{\prime}+\theta_{x t} g^{\prime}=\varphi_{2}^{\prime}, \\
\theta_{x t} f^{\prime}+\theta_{t t} g^{\prime}=\psi_{2}^{\prime} .
\end{gathered}
$$

So, we can rewrite the above system in the following form:

$$
\mathcal{A U}=\mathcal{F}
$$


where

$$
\mathcal{A}=\left(\begin{array}{cccccc}
1 & 0 & 0 & \alpha & -a & 0 \\
0 & 1 & \alpha & 0 & 0 & -k(0) \\
0 & 0 & g^{\prime} & 0 & f^{\prime} & 0 \\
g^{\prime} & 0 & f^{\prime} & 0 & 0 & 0 \\
0 & 0 & 0 & g^{\prime} & 0 & f^{\prime} \\
0 & g^{\prime} & 0 & f^{\prime} & 0 & 0
\end{array}\right), \quad \mathcal{U}=\left(\begin{array}{c}
v_{t t} \\
\theta_{t t} \\
v_{x t} \\
\theta_{x t} \\
v_{x x} \\
\theta_{x x}
\end{array}\right), \quad \mathcal{F}=\left(\begin{array}{c}
0 \\
f \\
\varphi_{1}^{\prime} \\
\psi_{1}^{\prime} \\
\varphi_{2}^{\prime} \\
\psi_{2}^{\prime}
\end{array}\right)
$$

After some calculations it is not difficult to see that

$$
\operatorname{det}(\mathcal{A})=-\left(f^{\prime}\right)^{4}+\left(k(0)+a+\alpha^{2}\right)\left(f^{\prime} g^{\prime}\right)^{2}-a k(0)\left(g^{\prime}\right)^{4}
$$

Therefore, a characteristic curve will satisfy

$$
(d x)^{4}-\left(k(0)+a+\alpha^{2}\right)(d t d x)^{2}+a k(0)(d t)^{4}=0,
$$

and since

$$
\left(k(0)+a+\alpha^{2}\right)^{2}-4 a k(0)=(k(0)-a)^{2}+2(k(0)+a) \alpha^{2}+\alpha^{4}>0,
$$

for any $\alpha$, we conclude that system (1.1)-(1.2) is of hyperbolic type.

3. Existence and regularity. In this section we prove the existence and regularity of weak solutions of the hyperbolic thermoelastic equation with memory. Let us introduce the following space:

$$
W=\left\{w \in H^{1}(0, L) ; w(0)=w(L)=0\right\} .
$$

To simplify our analysis let us define the binary operator

$$
k \square v(t)=\int_{0}^{t} k(t-\tau) \int_{0}^{L}|v(x, t)-v(x, \tau)|^{2} d x d \tau .
$$

Under this notation we have

Lemma 3.1. For any $v \in C\left([0, T] ; H^{1}(0, L)\right)$ and $\eta \in C^{1}(\mathbb{R})$,

$$
\begin{aligned}
\int_{0}^{L} \int_{0}^{t} \eta(t-\tau) v(x, \tau) d \tau v_{t}(x, t) d x=-\frac{1}{2} \eta(t) \int_{0}^{L}|v|^{2} d x & +\frac{1}{2} \eta^{\prime} \square v \\
& -\frac{1}{2} \frac{d}{d t}\left\{\eta \square v-\left(\int_{0}^{t} \eta d t\right) \int_{0}^{L}|v|^{2} d x\right\} .
\end{aligned}
$$

Proof. To show the above identity, it is enough to differentiate with respect to time the expression $\eta \square v$.

The definition of weak solution we use in this work is given as follows.

DEFINITION 3.1. We say that the pair $(u, \theta)$ is a weak solution of the system (1.1)(1.2) if

$$
\begin{aligned}
& u \in C([0, T] ; W) \cap C^{1}\left([0, T] ; L^{2}(0, L)\right), \\
& \theta \in C\left([0, T] ; L^{2}(0, L)\right), \\
& k * \theta \in L^{2}([0, T] ; W),
\end{aligned}
$$


and satisfies the following identities:

$$
\begin{aligned}
\int_{0}^{T} \int_{0}^{L} u \Phi_{t t} d x d t+a \int_{0}^{T} \int_{0}^{L} u_{x} \Phi_{x} d x d t-\alpha \int_{0}^{T} \int_{0}^{L} \theta \Phi_{x} d x d t \\
=-\int_{0}^{L} u_{0}(x) \Phi_{t}(x, 0) d x+\int_{0}^{L} u_{1}(x) \Phi(x, 0) d x \\
\quad-\int_{0}^{T} \int_{0}^{L} \theta \Phi_{t} d x d t+\int_{0}^{T} \int_{0}^{L} k * \theta_{x} \Phi_{x} d x d t-\alpha \int_{0}^{T} \int_{0}^{L} u_{t} \Phi_{x} d x d t \\
=\int_{0}^{L} \theta_{0}(x) \Phi(x, 0) d x
\end{aligned}
$$

for any $\Phi \in C^{2}([0, T] ; W)$ such that $\Phi(\cdot, T)=\Phi_{t}(\cdot, T)=0$.

Let us introduce the energy functions

$$
\begin{aligned}
& E(t ; v, \varphi):=\frac{1}{2} \int_{0}^{L}\left[\left|v_{t}\right|^{2}+a\left|v_{x}\right|^{2}+|\varphi|^{2}\right] d x, \\
& F(t ; v, \varphi):=\frac{1}{2} \int_{0}^{L}\left[\left|v_{t t}\right|^{2}+a\left|v_{x t}\right|^{2}+\left|\varphi_{t}\right|^{2}+k(t)\left|\varphi_{x}\right|^{2}-k^{\prime} \square \varphi_{x}\right] d x,
\end{aligned}
$$

and let us denote by $\left\{w_{j} \in W ; j \in \mathbb{N}\right\}$ an orthonormal basis of $W$ such that $-w_{j, x x}=$ $\lambda_{j} w_{j}$.

The following lemma will play an important role in the sequel.

Lemma 3.2. Let us suppose that $k \in L^{1}\left(\mathbb{R}^{+}\right)$is a strongly positive definite kernel satisfying $k^{\prime} \in L^{1}\left(\mathbb{R}^{+}\right)$; then we have

$$
\int_{0}^{t}|k * y(\tau)|^{2} d \tau \leq \beta_{0} K \int_{0}^{t} k * y(\tau) y(\tau) d \tau
$$

for any $y \in L_{\text {loc }}^{1}\left(\mathbb{R}^{+}\right)$where $K=|k|_{1}^{2}+4\left|k^{\prime}\right|_{1}^{2}$ and $\beta_{0}>0$ is such that the function $k(t)-\beta_{0} e^{-t}$ is a positive definite kernel.

Proof. See [15].

In these conditions we are able to prove the following theorem.

Theorem 3.1. Let us suppose that the initial data satisfies $\left(u_{0}, u_{1}, \theta_{0}\right) \in W \times L^{2} \times L^{2}$. Then there exists a unique weak solution for (1.1)-(1.2). Moreover, if we take the initial data

$$
u_{0} \in H^{2}(0, L) \cap W, \quad u_{1} \in W, \quad \theta_{0} \in W
$$

then the solution satisfies

$$
\begin{aligned}
& u \in C\left([0, T] ; H^{2}(0, L) \cap W\right) \cap C^{1}([0, T] ; W) \cap C^{2}\left([0, T] ; L^{2}(0, L)\right), \\
& \theta \in C([0, T] ; W) \cap C^{1}\left([0, T] ; L^{2}(0, L)\right) .
\end{aligned}
$$

Proof. Our starting point is to construct the Galerkin approximation $u^{m}$ and $\theta^{m}$ of the solution. Let us denote by

$$
u^{m}(\cdot, t)=\sum_{j=1}^{m} h_{j, m}(t) w_{j}(\cdot), \quad \theta^{m}(\cdot, t)=\sum_{j=1}^{m} g_{j, m}(t) w_{j}(\cdot),
$$


where the functions $g_{j, m}$ and $h_{j, m}$ are given by the solution of the approximated system,

$$
\begin{gathered}
\int_{0}^{L} u_{t t}^{m} w_{j} d x+a \int_{0}^{L} u_{x}^{m} w_{j, x} d x-\alpha \int_{0}^{L} \theta^{m} w_{j, x} d x=0, \quad j=1, \ldots, m, \\
\int_{0}^{L} \theta_{t}^{m} w_{j} d x+\int_{0}^{L} k * \theta_{x}^{m} w_{j, x} d x-\alpha \int_{0}^{L} u_{t}^{m} w_{j, x} d x=0, \quad j=1, \ldots, m, \\
u^{m}(\cdot, 0)=u_{0, m}, \quad u_{t}^{m}(\cdot, 0)=u_{1, m}, \quad \theta^{m}(\cdot, 0)=\theta_{0, m},
\end{gathered}
$$

and

$$
\begin{gathered}
u_{0, m}=\sum_{j=1}^{m}\left\{\int_{0}^{L} u_{0} w_{j} d x\right\} w_{j}, \quad u_{1, m}=\sum_{j=1}^{m}\left\{\int_{0}^{L} u_{1} w_{j} d x\right\} w_{j} \\
\theta_{0, m}=\sum_{j=1}^{m}\left\{\int_{0}^{L} \theta_{0} w_{j} d x\right\} w_{j} .
\end{gathered}
$$

The existence of the approximated solutions $u^{m}$ and $\theta^{m}$ is guaranteed by standard results on ordinary differential equations. Our next step is to show that the approximated solution remains bounded for any $m>0$. To this end, let us multiply equation (3.1) by $h_{j, m}^{\prime}$ and (3.2) by $g_{j, m}$. Summing the product result in $j$ we arrive at

$$
\frac{d}{d t} E\left(t ; u^{m}, \theta^{m}\right)=-\int_{0}^{L} k * \theta_{x}^{m} \theta_{x}^{m} d x .
$$

Integrating from 0 to $t$ the above relation and using Lemma 3.2 it follows that

$$
E\left(t ; u^{m}, \theta^{m}\right)+C_{0} \int_{0}^{t} \int_{0}^{L}\left|k * \theta_{x}^{m}\right|^{2} d x d \tau \leq E\left(0 ; u^{m}, \theta^{m}\right) .
$$

From our choice of $u_{0, m}, u_{1, m}$, and $\theta_{0, m}$ it follows that

$$
\begin{array}{ll}
\left(u_{x}^{m}, u_{t}^{m}, \theta^{m}\right) & \text { is bounded in } L^{\infty}\left(0, T ; L^{2}(0, L)\right), \\
k * \theta_{x}^{m} & \text { is bounded in } L^{2}\left(0, T ; L^{2}(0, L)\right) .
\end{array}
$$

Let us denote $U^{m}=u^{m}-u^{n}$ and $\Theta^{m}=\theta^{m}-\theta^{n}$ with $m>n$. We may rewrite the sequences $u^{n}$ and $\theta^{n}$ as

$$
u^{n}(\cdot, t)=\sum_{j=1}^{m} h_{j, n}(t) w_{j}(\cdot), \quad \theta^{n}(\cdot, t)=\sum_{j=1}^{m} g_{j, n}(t) w_{j}(\cdot),
$$

where

$$
h_{j, n}(t)=g_{j, n}(t)=0, \quad n<j \leq m .
$$

Using similar arguments as above we can show the corresponding inequality (3.3) to $\left\{U^{m}, \Theta^{m}\right\}$. This implies that $\left\{u^{m}\right\}$ and $\left\{\theta^{m}\right\}$ are Cauchy sequences in $C^{1}\left(0, T ; L^{2}(0, L)\right) \cap$ $C\left(0, T ; H^{1}(0, L)\right)$ and $C\left(0, T ; L^{2}(0, L)\right)$, respectively. Multiplying equations $(3.1)$ and (3.2) by $\varphi \in C^{1}([0, T])$ such that $\varphi(T)=\varphi_{t}(T)=0$ and performing an integration over 
$[0, T]$ we have

$$
\begin{aligned}
\int_{0}^{T} & \int_{0}^{L} u^{m} w_{j} \varphi_{t t} d x d t+a \int_{0}^{T} \int_{0}^{L} u_{x}^{m} w_{j, x} \varphi d x d t-\alpha \int_{0}^{T} \int_{0}^{L} \theta^{m} w_{j, x} \varphi d x d t \\
= & \int_{0}^{L} u_{1, m} w_{j} \varphi(0) d x+\int_{0}^{L} u_{0, m} w_{j} \varphi_{t}(0) d x \\
& -\int_{0}^{T} \int_{0}^{L} \theta^{m} w_{j} \varphi_{j} d x d t+\int_{0}^{T} \int_{0}^{L} k * \theta_{x}^{m} w_{j, x} \varphi d x d t-\alpha \int_{0}^{T} \int_{0}^{L} u_{t}^{m} w_{j, x} \varphi d x d t \\
= & \int_{0}^{L} \theta_{0, m} w_{j} \varphi(0) d x .
\end{aligned}
$$

From (3.4), (3.5) and using the density of the set $\left\{w_{j} \varphi_{j} ; j \in \mathbb{N}, \varphi_{j} \in C^{1}([0, T])\right\}$ in $C^{1}([0, T] ; W)$ we conclude that $(u, \theta)$ is a weak solution of the system (1.1)-(1.2), where $(u, \theta)$ are obtained by taking the limit when $m \rightarrow \infty$. The uniqueness follows by standard methods for hyperbolic equations. To show the regularity let us differentiate equations (3.1) and (3.2) with respect to time to get

$$
\begin{gathered}
\int_{0}^{L} u_{t t t}^{m} w_{j} d x+a \int_{0}^{L} u_{x t}^{m} w_{j, x} d x-\alpha \int_{0}^{L} \theta_{t}^{m} w_{j, x} d x=0 \\
\int_{0}^{L} \theta_{t t}^{m} w_{j} d x+k(0) \int_{0}^{L} \theta_{x}^{m} w_{j, x} d x+\int_{0}^{L} k^{\prime} * \theta_{x}^{m} w_{j, x} d x+\alpha \int_{0}^{L} u_{x t t}^{m} w_{j} d x=0 .
\end{gathered}
$$

Multiplying equation (3.6) by $h_{j, m}^{\prime \prime}$ and (3.7) by $g_{j, m}^{\prime}$ and using similar arguments as above our conclusion follows.

4. Uniform rate of decay. In this section we show that the solution of the system (1.1)-(1.2) decays exponentially as time goes to infinity. The method we use here is based on the construction of a functional $\mathcal{L}(t)$ equivalent to the first-order energy whose derivative is negative proportional to itself. Here we will assume that $k$ and $-k^{\prime}$ are positive definite kernels satisfying

$$
\begin{gathered}
k^{\prime}, k^{\prime \prime} \in C^{1}\left(\mathbb{R}_{+}\right), \\
\int_{0}^{T} k^{\prime} * \varphi \varphi d t \leq-C \int_{0}^{T} k * \varphi \varphi d t .
\end{gathered}
$$

The above inequality is satisfied for any function $k$ that is the sum of exponential functions with varying rates of decay. Since the kernel $k$ decays exponentially, we take $\gamma>0$ small enough such that there exists $\varepsilon>0$ satisfying

$$
\hat{k}(t):=e^{\gamma t} k(t) \leq C e^{-\varepsilon t} .
$$

Let us denote $v(x, t)=u(x, t) e^{\gamma t}$ and $\varphi(x, t)=\theta(x, t) e^{\gamma t}$. Then

$$
\begin{aligned}
v_{t} & =u_{t} e^{\gamma t}+\gamma u e^{\gamma t}=u_{t} e^{\gamma t}+\gamma v, \\
v_{t t} & =u_{t t} e^{\gamma t}+\gamma u_{t} e^{\gamma t}+\gamma u_{t} e^{\gamma t}+\gamma^{2} u e^{\gamma t}=u_{t t} e^{\gamma t}+2 \gamma v_{t}-\gamma^{2} v, \\
\varphi_{t} & =\theta_{t} e^{\gamma t}+\gamma \varphi, \\
\hat{k} * \varphi_{x x} & =e^{\gamma t}\left(k * \theta_{x x}\right) .
\end{aligned}
$$


From the above identities it is easy to see that the pair $(v, \varphi)$ satisfies

$$
\begin{aligned}
v_{t t}-a v_{x x}+\alpha \varphi_{x} & =R, \\
\varphi_{t}-\hat{k} * \varphi_{x x}+\alpha v_{x t} & =S,
\end{aligned}
$$

where $R$ and $S$ are given by

$$
\begin{aligned}
R & :=2 \gamma v_{t}-\gamma^{2} v, \\
S & :=\gamma \varphi+\alpha \gamma v_{x}
\end{aligned}
$$

with $v$ and $\varphi$ satisfying the same boundary conditions and initial values as in system (1.1)-(1.2). Let us introduce the energy function

$$
E(t ; v, \varphi):=\frac{1}{2} \int_{0}^{L}\left[\left|v_{t}\right|^{2}+a\left|v_{x}\right|^{2}+|\varphi|^{2}\right] d x
$$

So, to show the exponential decay of the couple $(u, \theta)$ it is enough to show that $E(T, v, \varphi)$ is bounded. To this end, we start with the following Lemma.

LEMmA 4.1. Let us suppose that the initial data satisfy

$$
u_{0} \in H^{2}(0, L) \cap W, \quad u_{1} \in W, \quad \theta_{0} \in W
$$

Then we have

$$
\frac{d}{d t} E(t ; v, \varphi) \leq-\int_{0}^{L} \hat{k} * \varphi_{x} \varphi_{x} d x+c \gamma \int_{0}^{L}\left(\left|v_{t}\right|^{2}+\left|v_{x}\right|^{2}+|\varphi|^{2}\right) d x
$$

Proof. Multiplying equation (4.3) by $v_{t}$ and equation (4.4) by $\varphi$ and integrating from 0 to $L$, we get

$$
\frac{d}{d t} E(t ; v, \varphi)=-\int_{0}^{L} \hat{k} * \varphi_{x} \varphi_{x} d x+\int_{0}^{L}\left(R v_{t}+S \varphi\right) d x
$$

After applying Hölder's inequality and Young's inequality we have

$$
\begin{aligned}
\left|\int_{0}^{L} R v_{t} d x\right| & \leq \frac{5 \gamma}{2} \int_{0}^{L}\left|v_{t}\right|^{2} d x+\frac{c_{p} \gamma^{3}}{2} \int_{0}^{L}\left|v_{x}\right|^{2} d x \\
\left|\int_{0}^{L} S \varphi d x\right| & \leq \frac{3 \gamma}{2} \int_{0}^{L}|\varphi|^{2} d x+\frac{\alpha^{2} \gamma}{2} \int_{0}^{L}\left|v_{x}\right|^{2} d x
\end{aligned}
$$

Thereby, our conclusion follows.

To get $-\int_{0}^{L}|\varphi|^{2} d x$ and $-\int_{0}^{L}\left|v_{x}\right|^{2} d x$ we will use the following lemma (note that $\hat{k}(0)=k(0))$. 
LEMmA 4.2. With the same hypotheses as Lemma 4.1 we have

$$
\begin{aligned}
-\frac{d}{d t} \int_{0}^{L} \hat{k} * \varphi \varphi d x \leq & -\frac{\hat{k}(0)}{4} \int_{0}^{L}|\varphi|^{2} d x+C \int_{0}^{L}\left|\hat{k} * \varphi_{x}\right|^{2} d x+C \int_{0}^{L}\left|\hat{k}^{\prime} * \varphi_{x}\right|^{2} d x \\
& -\alpha \int_{0}^{L} v_{t} \hat{k} * \varphi_{x} d x-\int_{0}^{L} S(\hat{k} * \varphi) d x \\
\frac{d}{d t} \int_{0}^{L} v v_{t} d x \leq & -\frac{a}{2} \int_{0}^{L}\left|v_{x}\right|^{2} d x+\int_{0}^{L}\left|v_{t}\right|^{2} d x+\frac{\alpha^{2}}{2 a} \int_{0}^{L}|\varphi|^{2} d x \\
& +\gamma c \int_{0}^{L}\left(\left|v_{t}\right|^{2}+\left|v_{x}\right|^{2}\right) d x .
\end{aligned}
$$

Proof. Multiplying equation (4.4) by $-\hat{k} * \varphi$ and integrating from 0 to $L$ we have

$$
\int_{0}^{L}\left(\varphi_{t}-\hat{k} * \varphi_{x x}+\alpha v_{x t}\right)(-\hat{k} * \varphi) d x=-\int_{0}^{L} S(\hat{k} * \varphi) d x .
$$

Note that

$$
\begin{aligned}
-\int_{0}^{L} \varphi_{t} \hat{k} * \varphi d x & =\frac{d}{d t} \int_{0}^{L} \varphi(-\hat{k} * \varphi) d x+\hat{k}(0) \int_{0}^{L}|\varphi|^{2} d x+\int_{0}^{L} \hat{k}^{\prime} * \varphi \varphi d x, \\
\int_{0}^{L} \hat{k} * \varphi_{x x}(\hat{k} * \varphi) d x & =-\int_{0}^{L}\left|\hat{k} * \varphi_{x}\right|^{2} d x, \\
\alpha \int_{0}^{L} v_{x t}(-\hat{k} * \varphi) d x & =\alpha \int_{0}^{L} v_{t} \hat{k} * \varphi_{x} d x .
\end{aligned}
$$

On the other hand, from (4.3) we have

$$
\begin{aligned}
\frac{d}{d t} \int_{0}^{L} v v_{t} d x= & \int_{0}^{L}\left|v_{t}\right|^{2} d x+\int_{0}^{L} v v_{t t} d x \\
= & \int_{0}^{L}\left|v_{t}\right|^{2} d x+a \int_{0}^{L} v v_{x x} d x-\alpha \int_{0}^{L} v \varphi_{x} d x+\int_{0}^{L} R v d x \\
\leq & \int_{0}^{L}\left|v_{t}\right|^{2} d x-\frac{a}{2} \int_{0}^{L}\left|v_{x}\right|^{2} d x+\frac{\alpha^{2}}{2 a} \int_{0}^{L}|\varphi|^{2} d x \\
& +\gamma\left(1+c_{p}\right) \int_{0}^{L}\left(\left|v_{t}\right|^{2}+\left|v_{x}\right|^{2}\right) d x
\end{aligned}
$$

The proof is now complete.

Let us introduce the multipliers $q$ and $w$ given by

$$
\begin{aligned}
-q_{x x} & =v_{t} & & \text { in }(0, L) \times \mathbb{R}^{+}, \\
q(0, t) & =q(L, t)=0 & & \text { in } \mathbb{R}^{+}, \\
-w_{x x} & =\varphi-\bar{\varphi} & & \text { in }(0, L) \times \mathbb{R}^{+}, \\
w_{x}(0, t) & =w_{x}(L, t)=0 & & \text { in } \mathbb{R}^{+},
\end{aligned}
$$


where $\bar{\varphi}=\frac{1}{L} \int_{0}^{L} \varphi d x$. We easily get

$$
\begin{gathered}
\left\|q_{x}\right\|_{H^{1}}^{2} \leq\left(c_{p}+1\right) \int_{0}^{L}\left|v_{t}\right|^{2} d x \\
\int_{0}^{L}\left|w_{x}\right|^{2} d x \leq c \int_{0}^{L}|\varphi|^{2} d x .
\end{gathered}
$$

In this condition we have

Lemma 4.3. With the same hypotheses as Lemma 4.1 we have that for any $(v, \varphi)$ and any $\delta>0$, there exist $C_{\delta}>0$ such that

$$
\int_{0}^{T}\left\|q_{x}(\cdot, \tau)\right\|_{H^{\frac{3}{4}}}^{2} d t \leq \delta \int_{0}^{T} \int_{0}^{L}\left(\left|v_{t}\right|^{2}+|\varphi|^{2}+\left|v_{x}\right|^{2}\right) d x d t+C_{\delta} \int_{0}^{T} \int_{0}^{L}\left|\hat{k} * \varphi_{x}\right|^{2} d x d t
$$

Proof. We reason by contradiction. Let us suppose that there exists a sequence $\left(v^{\nu}, \varphi^{\nu}\right)$ and $\delta_{0}>0$ such that

$$
\int_{0}^{T}\left\|q_{x}^{\nu}(\cdot, \tau)\right\|_{H^{\frac{3}{4}}}^{2} d t \geq \delta_{0} \int_{0}^{T} \int_{0}^{L}\left(\left|v_{t}^{\nu}\right|^{2}+\left|\varphi^{\nu}\right|^{2}+\left|v_{x}^{\nu}\right|^{2}\right) d x d t+\nu \int_{0}^{T} \int_{0}^{L}\left|\hat{k} * \varphi_{x}^{\nu}\right|^{2} d x d t
$$

where

$$
\int_{0}^{T}\left\|q_{x}^{\nu}(\cdot, \tau)\right\|_{H^{\frac{3}{4}}}^{2} d t=1
$$

It follows that

$$
v_{t}^{\nu}, \varphi^{\nu}, v_{x}^{\nu} \text { are bounded in } L^{\infty}\left(0, T ; L^{2}(0, L)\right)
$$

and also that

$$
\hat{k} * \varphi_{x}^{\nu} \rightarrow 0 \quad \text { strong in } L^{2}\left(0, T ; L^{2}(0, L)\right) .
$$

From (4.14) we have that there exist $\left(v_{t}, \varphi, v_{x}\right)$ such that

$$
\left(v_{t}^{\nu}, \varphi^{\nu}, v_{x}^{\nu}\right) \rightarrow\left(v_{t}, \varphi, v_{x}\right) \text { in }\left[L^{2}\left(0, T ; L^{2}(0, L)\right)\right]^{3} .
$$

The above convergence together with (4.15) implies

$$
\hat{k} * \varphi_{x}^{\nu} \rightarrow \hat{k} * \varphi_{x}=0 .
$$

Since $k * \varphi$ satisfies Dirichlet boundary conditions, we have that $k * \varphi=0$ and, using Volterra's resolvent, we conclude that $\varphi=0$. On the other hand, since $-q_{x x}^{\nu}=v_{t}^{\nu}$ from (4.14) it follows that

$$
q_{x}^{\nu}, q_{t}^{\nu} \text { is bounded in } L^{2}\left(0, T ; H^{1}(0, L)\right) .
$$

Applying Lions-Aubin's Theorem (see [9], Theorem 5.1, p. 58) we conclude that

$$
q_{x}^{\nu} \rightarrow q_{x} \quad \text { strong in } L^{2}\left(0, T ; H^{\frac{3}{4}}(0, L)\right) .
$$

From (4.13) we have

$$
\int_{0}^{T}\left\|q_{x}(\cdot, \tau)\right\|_{H^{\frac{3}{4}}}^{2} d t=1
$$

Using (4.4) and since $v$ satisfies Dirichlet's boundary condition we have that

$$
v_{x t}=\gamma v_{x} \Rightarrow v_{x}(x, t)=v_{x}(x, 0) e^{\gamma t} \Rightarrow v(x, t)=v(x, 0) e^{\gamma t} .
$$


Using (4.3) we have that

$$
-a v_{x x}=0 .
$$

Since $v$ satisfies Dirichlet's condition we conclude that $v=0$. Therefore we have that

$$
\left(v_{t}^{\nu}, \varphi^{\nu}, v_{x}^{\nu}\right) \rightarrow 0 \quad \text { in } L^{2}\left(0, T ; L^{2}(0, L)^{3}\right) .
$$

Finally, since $-q_{x x}^{\nu}=v_{t}^{\nu}$ from (4.16) we conclude that $q_{x}=0$. But this is contradictory with (4.17). The proof is now complete.

LEMmA 4.4. With the same hypotheses as Lemma 4.1 we have that

$$
\begin{aligned}
\frac{d}{d t} \int_{0}^{L} q_{x} \varphi d x \leq & -\frac{\alpha}{2} \int_{0}^{L}\left|v_{t}\right|^{2} d x+\frac{\alpha a}{32} \int_{0}^{L}\left|v_{x}\right|^{2} d x+\left(\frac{8 \alpha}{a}+\alpha\right) \int_{0}^{L}|\varphi|^{2} d x \\
& +\frac{\alpha L}{8 \hat{k}(0)}\left\{\left|\hat{k} * \varphi_{x}(L, t)\right|^{2}+\left|\hat{k} * \varphi_{x}(0, t)\right|^{2}\right\}+\frac{8 \omega_{1}^{2} \hat{k}(0)}{\alpha L}\left\|q_{x}(\cdot, t)\right\|_{H^{\frac{3}{4}}}^{2} \\
& +\frac{1}{2 \alpha} \int_{0}^{L}\left|\hat{k} * \varphi_{x}\right|^{2} d x+c_{1} \gamma \int_{0}^{L}\left(\left|v_{t}\right|^{2}+|\varphi|^{2}+\left|v_{x}\right|^{2}\right) d x
\end{aligned}
$$

where $\omega_{1}$ is the embedding constant of $H^{\frac{3}{4}}(0, L) \hookrightarrow L^{\infty}(0, L)$ and $c_{1}$ is a constant that does not depend on $\gamma$.

Proof. Note that

$$
\begin{aligned}
\frac{d}{d t} \int_{0}^{L} q_{x} \varphi d x & =\int_{0}^{L} q_{x t} \varphi d x+\int_{0}^{L} q_{x} \varphi_{t} d x \\
& =\underbrace{\int_{0}^{L} q_{x t} \varphi d x}_{I_{1}}+\underbrace{\int_{0}^{L} q_{x} \hat{k} * \varphi_{x x} d x}_{I_{2}}+\underbrace{\int_{0}^{L} q_{x} v_{x t} d x}_{I_{3}}+\underbrace{\int_{0}^{L} q_{x} S d x}_{I_{4}} .
\end{aligned}
$$

Recalling the definitions of $w, v$ and $q$ we have

$$
\begin{aligned}
I_{1} & =\int_{0}^{L} q_{x t}(\varphi-\bar{\varphi}) d x=-\int_{0}^{L} q_{x t} w_{x x} d x \\
& =\int_{0}^{L} q_{x x t} w_{x} d x=-\int_{0}^{L} v_{t t} w_{x} d x .
\end{aligned}
$$

Using equation (4.3) we arrive at

$$
\begin{aligned}
I_{1} & =-a \int_{0}^{L} v_{x x} w_{x} d x+\alpha \int_{0}^{L} \varphi_{x} w_{x} d x-\int_{0}^{L} R w_{x} d x \\
& =a \int_{0}^{L} v_{x} w_{x x} d x-\alpha \int_{0}^{L} \varphi w_{x x} d x-\int_{0}^{L} R w_{x} d x \\
& =-a \int_{0}^{L} v_{x}(\varphi-\bar{\varphi}) d x+\alpha \int_{0}^{L} \varphi(\varphi-\bar{\varphi}) d x-\int_{0}^{L} R w_{x} d x \\
& =-a \int_{0}^{L} v_{x} \varphi d x+\alpha \int_{0}^{L}|\varphi|^{2} d x-\alpha\left(\int_{0}^{L} \varphi d x\right)^{2}-\int_{0}^{L} R w_{x} d x .
\end{aligned}
$$


Since

$$
\begin{aligned}
\int_{0}^{L} R w_{x} d x & =\int_{0}^{L}\left(2 \gamma v_{t}-\gamma^{2} v\right) w_{x} d x \\
& \leq c \gamma \int_{0}^{L}\left(\left|v_{t}\right|^{2}+|\varphi|^{2}+\left|v_{x}\right|^{2}\right) d x \\
\left|\alpha \int_{0}^{L} v_{x} \varphi d x\right| & \leq \frac{\alpha a}{32} \int_{0}^{L}\left|v_{x}\right|^{2} d x+\frac{8 \alpha}{a} \int_{0}^{L}|\varphi|^{2} d x
\end{aligned}
$$

We conclude that

$$
I_{1} \leq \frac{\alpha a}{32} \int_{0}^{L}\left|v_{x}\right|^{2} d x+\left(\frac{8 \alpha}{a}+\alpha\right) \int_{0}^{L}|\varphi|^{2} d x+c \gamma \int_{0}^{L}\left(\left|v_{t}\right|^{2}+|\varphi|^{2}+\left|v_{x}\right|^{2}\right) d x .
$$

Now we consider the term $I_{2}$ :

$$
\begin{aligned}
I_{2} & =\left.q_{x} \hat{k} * \varphi_{x}\right|_{0} ^{L}-\int_{0}^{L} q_{x x} \hat{k} * \varphi_{x} d x \\
& \leq\left\|q_{x}(\cdot, t)\right\|_{L_{\infty}}\left\{\left|\hat{k} * \varphi_{x}(L, t)\right|+\left|\hat{k} * \varphi_{x}(0, t)\right|\right\}+\int_{0}^{L} v_{t} \hat{k} * \varphi_{x} d x \\
& \leq \omega_{1}\left\|q_{x}(\cdot, t)\right\|_{H^{\frac{3}{4}}}\left\{\left|\hat{k} * \varphi_{x}(L, t)\right|+\left|\hat{k} * \varphi_{x}(0, t)\right|\right\}+\int_{0}^{L} v_{t} \hat{k} * \varphi_{x} d x .
\end{aligned}
$$

Applying Hölder's inequality and Young's inequality in the last expression we have

$$
\begin{aligned}
I_{2} \leq & \frac{\omega_{1}^{2} 8 \hat{k}(0)}{\alpha L}\left\|q_{x}(\cdot, t)\right\|_{H^{\frac{3}{4}}}^{2}+\frac{\alpha L}{8 \hat{k}(0)}\left\{\left|\hat{k} * \varphi_{x}(L, t)\right|^{2}+\left|\hat{k} * \varphi_{x}(0, t)\right|^{2}\right\} \\
& +\frac{\alpha}{2} \int_{0}^{L}\left|v_{t}\right|^{2} d x+\frac{1}{2 \alpha} \int_{0}^{L}\left|\hat{k} * \varphi_{x}\right|^{2} d x .
\end{aligned}
$$

Recalling the definition of $q$ we conclude that $I_{3}$ satisfies

$$
I_{3}=-\alpha \int_{0}^{L}\left|v_{t}\right|^{2} d x
$$

Finally, we have that

$$
I_{4}=\int_{0}^{L} q_{x}\left(\gamma \varphi+\alpha \gamma v_{x}\right) d x \leq c \gamma \int_{0}^{L}\left(\left|v_{t}\right|^{2}+|\varphi|^{2}+\left|v_{x}\right|^{2}\right) d x
$$

Taking the summation of the functions $I_{i}$ our conclusion follows.

One of the main difficulties in proving the exponential decay is to estimate the pointwise terms $\hat{k} * \varphi_{x}(0, t)$ and $\hat{k} * \varphi_{x}(L, t)$. We overcome this problem using the following lemma. 
LEMma 4.5. With the same hypotheses as Lemma 4.1 we have that

$$
\begin{aligned}
\frac{d}{d t} \mathcal{T}_{1}(t) \leq & \hat{k}(0) \int_{0}^{L}|\varphi|^{2} d x-\frac{L}{4}\left\{\left|\hat{k} * \varphi_{x}(L, t)\right|^{2}+\left|\hat{k} * \varphi_{x}(0, t)\right|^{2}\right\} \\
& +C \int_{0}^{L}\left|\hat{k} * \varphi_{x}\right|^{2} d x+\frac{\hat{k}(0)}{2} \int_{0}^{L}\left|v_{t}\right|^{2} d x+\hat{k}(0) a \int_{0}^{L}\left|v_{x}\right|^{2} d x \\
& -\frac{\hat{k}(0) a L}{4}\left\{\left|v_{x}(L, t)\right|^{2}+\left|v_{x}(0, t)\right|^{2}\right\}+c \gamma \int_{0}^{L}\left(\left|v_{t}\right|^{2}+|\varphi|^{2}+\left|v_{x}\right|^{2}+\left|\hat{k} * \varphi_{x}\right|^{2}\right) d x
\end{aligned}
$$

where $\mathcal{T}_{1}(t)=-\int_{0}^{L}\left(x-\frac{L}{2}\right)\left\{\varphi \hat{k} * \varphi_{x}+\alpha v_{k} \hat{k} * \varphi_{x}+\hat{k}(0) v_{x} v_{t}\right\} d x$.

Proof. Multiplying (4.4) by $\left(x-\frac{L}{2}\right) \hat{k} * \varphi_{x}$ we get

$$
\begin{aligned}
\underbrace{\int_{0}^{L} \varphi_{t}\left(x-\frac{L}{2}\right) \hat{k} * \varphi_{x} d}_{I_{5}} & \underbrace{-\int_{0}^{L} \hat{k} * \varphi_{x x}\left(x-\frac{L}{2}\right) \hat{k} * \varphi_{x} d x}_{I_{6}} \\
\times & \underbrace{\int_{0}^{L} v_{x t}\left(x-\frac{L}{2}\right) \hat{k} * \varphi_{x} d x}_{I_{7}}=\int_{0}^{L} S\left(x-\frac{L}{2}\right) \hat{k} * \varphi_{x} d x .
\end{aligned}
$$

Now we consider each term $I_{i}$. We have

$$
\begin{aligned}
I_{5}= & \frac{d}{d t} \int_{0}^{L} \varphi\left(x-\frac{L}{2}\right) \hat{k} * \varphi_{x} d x-\int_{0}^{L} \varphi\left(x-\frac{L}{2}\right) \hat{k}(0) \varphi_{x} d x-\int_{0}^{L} \varphi\left(x-\frac{L}{2}\right) \hat{k}^{\prime} * \varphi_{x} d x \\
= & \frac{d}{d t} \int_{0}^{L} \varphi\left(x-\frac{L}{2}\right) \hat{k} * \varphi_{x} d x-\frac{\hat{k}(0)}{2} \int_{0}^{L}\left(x-\frac{L}{2}\right) \frac{d}{d x}|\varphi|^{2} d x \\
& -\int_{0}^{L} \varphi\left(x-\frac{L}{2}\right) \hat{k}^{\prime} * \varphi_{x} d x \\
= & \frac{d}{d t} \int_{0}^{L} \varphi\left(x-\frac{L}{2}\right) \hat{k} * \varphi_{x} d x+\frac{\hat{k}(0)}{2} \int_{0}^{L}|\varphi|^{2} d x-\int_{0}^{L} \varphi\left(x-\frac{L}{2}\right) \hat{k}^{\prime} * \varphi_{x} d x, \\
I_{6}= & -\frac{1}{2} \int_{0}^{L}\left(x-\frac{L}{2}\right) \frac{d}{d x}\left|\hat{k} * \varphi_{x}\right|^{2} d x \\
= & -\frac{L}{4}\left\{\left|\hat{k} * \varphi_{x}(L, t)\right|^{2}+\left|\hat{k} * \varphi_{x}(0, t)\right|^{2}\right\}+\frac{1}{2} \int_{0}^{L}\left|\hat{k} * \varphi_{x}\right|^{2} d x, \\
I_{7}= & \alpha \frac{d}{d t} \int_{0}^{L} v_{x}\left(x-\frac{L}{2}\right) \hat{k} * \varphi_{x} d x-\underbrace{\alpha \int_{x}^{L}\left(x-\frac{L}{2}\right) \hat{k}(0) \varphi_{x} d x}_{I_{8}} \\
& -\alpha \int_{0}^{L} v_{x}\left(x-\frac{L}{2}\right) \hat{k}^{\prime} * \varphi_{x} d x .
\end{aligned}
$$


From (4.3) we have

$$
\begin{aligned}
I_{8}= & \hat{k}(0) \int_{0}^{L} v_{x}\left(x-\frac{L}{2}\right) v_{t t} d x-\hat{k}(0) a \int_{0}^{L} v_{x}\left(x-\frac{L}{2}\right) v_{x x} d x \\
& -\hat{k}(0) \int_{0}^{L} v_{x}\left(x-\frac{L}{2}\right) R d x \\
= & \hat{k}(0) \frac{d}{d t} \int_{0}^{L} v_{x}\left(x-\frac{L}{2}\right) v_{t} d x-\frac{\hat{k}(0)}{2} \int_{0}^{L}\left(x-\frac{L}{2}\right) \frac{d}{d x}\left|v_{t}\right|^{2} d x \\
& -\frac{\hat{k}(0) a}{2} \int_{0}^{L}\left(x-\frac{L}{2}\right) \frac{d}{d x}\left|v_{x}\right|^{2} d x-\hat{k}(0) \int_{0}^{L} v_{x}\left(x-\frac{L}{2}\right) R d x \\
= & \hat{k}(0) \frac{d}{d t} \int_{0}^{L} v_{x}\left(x-\frac{L}{2}\right) v_{t} d x+\frac{\hat{k}(0)}{2} \int_{0}^{L}\left|v_{t}\right|^{2} d x \\
& -\frac{\hat{k}(0) a L}{4}\left\{\left|v_{x}(L, t)\right|^{2}+\left|v_{x}(0, t)\right|^{2}\right\} \\
& +\frac{\hat{k}(0) a}{2} \int_{0}^{L}\left|v_{x}\right|^{2} d x-\hat{k}(0) \int_{0}^{L} v_{x}\left(x-\frac{L}{2}\right) R d x .
\end{aligned}
$$

On the other hand, we have

$$
\begin{gathered}
\left|\alpha \int_{0}^{L} v_{x}\left(x-\frac{L}{2}\right) \hat{k}^{\prime} * \varphi_{x} d x\right| \leq \frac{\hat{k}(0) a}{2} \int_{0}^{L}\left|v_{x}\right|^{2} d x+\frac{L^{2} \delta_{0}}{8 a \hat{k}(0)} \int_{0}^{L}\left|\hat{k} * \varphi_{x}\right|^{2} d x, \\
\left|\int_{0}^{L} \varphi\left(x-\frac{L}{2}\right) \hat{k}^{\prime} * \varphi_{x} d x\right| \leq \frac{\hat{k}(0)}{2} \int_{0}^{L}|\varphi|^{2} d x+C \int_{0}^{L}\left|\hat{k} * \varphi_{x}\right|^{2} d x .
\end{gathered}
$$

Using the above inequalities in (4.21) our conclusion follows.

The exponential decay is summarized in the following theorem.

THEOREM 4.1. Under the same hypotheses as Lemma 4.1, there exist positive constants $C_{0}$ and $\gamma$ such that

$$
E(t ; u, \theta) \leq C_{0} E(0 ; u, \theta) c^{-\gamma t} .
$$

Proof. Consider $\epsilon=\frac{\alpha}{4 \hat{k}(0)}$. Then $\epsilon \hat{k}(0)<\frac{\alpha}{16}$. From Lemmas 4.4 and 4.5 we have

$$
\begin{aligned}
\frac{d}{d t}\left\{\int_{0}^{L} q_{x} \varphi d x+\epsilon \mathcal{T}_{1}(t)\right\} \leq & -\frac{3 \alpha}{8} \int_{0}^{L}\left|v_{t}\right|^{2} d x+\frac{\alpha a}{16} \int_{0}^{L}\left|v_{x}\right|^{2} d x \\
& +\frac{8 \omega_{1}^{2} \hat{k}(0)}{\alpha L}\left\|q_{x}(\cdot, t)\right\|_{H^{\frac{3}{4}}}^{2}+\left(\frac{8 \alpha}{a}+\frac{1}{\alpha}+\epsilon \hat{k}(0)\right) \int_{0}^{L}|\varphi|^{2} d x \\
& +C \int_{0}^{L}\left|\hat{k} * \varphi_{x}\right|^{2} d x \\
& +c \gamma \int_{0}^{L}\left(\left|v_{t}\right|^{2}+|\varphi|+\left|v_{x}\right|^{2}+\left|\hat{k} * \varphi_{x}\right|^{2}\right) d x
\end{aligned}
$$


where $C=c\left(\alpha, L, \hat{k}(0), \delta_{0}\right)$. Let us introduce the functional

$$
\mathcal{E}_{1}(t)=\int_{0}^{L} q_{x} \varphi d x+\epsilon \mathcal{T}_{1}(t)+\frac{\alpha}{4} \int_{0}^{L} v v_{t} d x .
$$

From inequalities (4.22) and (4.7) we get

$$
\begin{aligned}
\frac{d}{d t} \mathcal{E}_{1}(t) \leq & -\frac{\alpha}{8} \int_{0}^{L}\left|v_{t}\right|^{2} d x-\frac{\alpha a}{16} \int_{0}^{L}\left|v_{x}\right|^{2} d x+\mu_{0} \int_{0}^{L}|\varphi|^{2} d x \\
& +C \int_{0}^{L}\left|\hat{k} * \varphi_{x}\right|^{2} d x+\frac{8 \omega_{1}^{2} \hat{k}(0)}{\alpha L}\left\|q_{x}(\cdot, t)\right\|_{H^{\frac{3}{4}}}^{2} \\
& +c \gamma \int_{0}^{L}\left(\left|v_{t}\right|^{2}+|\varphi|^{2}+\left|v_{x}\right|^{2}+\left|\hat{k} * \varphi_{x}\right|^{2}\right) d x,
\end{aligned}
$$

where $\mu_{0}=\left\{\frac{8 \alpha}{a}+\frac{1}{\alpha}+\epsilon \hat{k}(0)+\frac{\alpha^{3}}{8 a}\right\}$. Note that

$$
\left|\alpha \int_{0}^{L} v_{t} \hat{k} * \varphi_{x} d x\right| \leq \frac{\hat{k}(0) \alpha}{80 \mu_{0}} \int_{0}^{L}\left|v_{t}\right|^{2} d x+\frac{20 \mu_{0} \alpha}{\hat{k}(0)} \int_{0}^{L}\left|\hat{k} * \varphi_{x}\right|^{2} d x .
$$

Using the above estimate in (4.6) from Lemma 4.2 it follows that

$$
\begin{aligned}
-\frac{d}{d t} \int_{0}^{L} \hat{k} * \varphi \varphi & d x \leq-\frac{\hat{k}(0)}{4} \int_{0}^{L}|\varphi|^{2} d x+\frac{\hat{k}(0) \alpha}{80 \mu_{0}} \int_{0}^{L}\left|v_{t}\right|^{2} d x \\
& +C \int_{0}^{L}\left|\hat{k} * \varphi_{x}\right|^{2} d x+c \gamma \int_{0}^{L}\left(\left|v_{t}\right|^{2}+\left|v_{x}\right|^{2}+|\varphi|^{2}+\left|\hat{k} * \varphi_{x}\right|^{2}\right) d x .
\end{aligned}
$$

Let us write

$$
\mathcal{F}(t)=\mathcal{E}_{1}(t)-\frac{5 \mu_{0}}{\hat{k}(0)} \int_{0}^{L} \hat{k} * \varphi \varphi d x .
$$

From (4.23) and (4.24) we get

$$
\begin{aligned}
\frac{d}{d t} \mathcal{F}(t) \leq- & k_{0} \int_{0}^{L}\left(|\varphi|^{2}+\left|v_{t}\right|^{2}+\left|v_{x}\right|^{2}\right) d x+C \int_{0}^{L}\left|\hat{k} * \varphi_{x}\right|^{2} d x \\
& +\frac{8 \omega_{1}^{2} \hat{k}(0)}{\alpha L}\left\|q_{x}(\cdot, t)\right\|_{H^{\frac{3}{4}}}^{2}+\gamma c \int_{0}^{L}\left(\left|v_{t}\right|^{2}+|\varphi|^{2}+\left|v_{x}\right|^{2}+\left|\hat{k} * \varphi_{x}\right|^{2}\right) d x
\end{aligned}
$$

where $k_{0}=\min \left\{\frac{\mu_{0}}{4}, \frac{\alpha}{16}, \frac{\alpha a}{16}\right\}$. Finally, we consider the functional

$$
\mathcal{L}(t)=\mathcal{F}(t)+N E(t ; v, \varphi) .
$$

From Lemma 4.1 and inequality (4.25) it follows that

$$
\begin{aligned}
\frac{d}{d t} \mathcal{L}(t) \leq & -k_{0} \int_{0}^{L}|\varphi|^{2}+\left|v_{x}\right|^{2}+\left|v_{t}\right|^{2} d x+C \int_{0}^{L}\left|\hat{k} * \varphi_{x}\right|^{2} d x \\
& +\frac{8 \omega_{1}^{2} \hat{k}(0)}{\alpha L}\left\|q_{x}(\cdot, t)\right\|_{H^{\frac{3}{4}}}^{2}-N \int_{0}^{L} \hat{k} * \varphi_{x} \varphi_{x} d x+N c \gamma \int_{0}^{L}\left(\left|v_{t}\right|^{2}+\left|v_{x}\right|^{2}+|\varphi|^{2}\right) d x \\
& +c \gamma \int_{0}^{L}\left(\left|v_{t}\right|^{2}+\left|v_{x}\right|^{2}+|\varphi|^{2}+\left|\hat{k} * \varphi_{x}\right|^{2}\right) d x .
\end{aligned}
$$


Integrating from 0 to $T$ the above relation, using Lemma 3.2 and the inequality

$$
\int_{0}^{T} \int_{0}^{L}\left|k^{\prime} * \varphi\right|^{2} d x d t \leq-C \int_{0}^{T} \int_{0}^{L} k^{\prime} * \varphi \varphi d x d t \leq c_{0} \int_{0}^{T} \int_{0}^{L} k * \varphi \varphi d x d t
$$

it follows that

$$
\begin{aligned}
\mathcal{L}(T)-\mathcal{L}(0) \leq & -k_{0} \int_{0}^{T} \int_{0}^{L}\left(|\varphi|^{2}+\left|v_{x}\right|^{2}+\left|v_{t}\right|^{2}\right) d x d \tau \\
& -\left(\frac{N}{K \beta_{0}}-C\right) \int_{0}^{T} \int_{0}^{L}\left|\hat{k} * \varphi_{x}\right|^{2} d x d \tau \\
& +\frac{8 \omega_{1}^{2} \hat{k}(0)}{\alpha L} \int_{0}^{T}\left\|q_{x}(\cdot, \tau)\right\|_{H^{\frac{3}{4}}}^{2} d \tau \\
& +N c \gamma \int_{0}^{T} \int_{0}^{L}\left(\left|v_{t}\right|^{2}+\left|v_{x}\right|^{2}+|\varphi|^{2}\right) d x d \tau \\
& +c \gamma \int_{0}^{T} \int_{0}^{L}\left(\left|v_{t}\right|^{2}+\left|v_{x}\right|^{2}+|\varphi|^{2}+\left|\hat{k} * \varphi_{x}\right|^{2}\right) d x d \tau .
\end{aligned}
$$

From Lemma 4.3 we have that

$$
\begin{aligned}
\mathcal{L}(T)-\mathcal{L}(0) \leq & -k_{0} \int_{0}^{T} \int_{0}^{L}\left(|\varphi|^{2}+\left|v_{x}\right|^{2}+\left|v_{t}\right|^{2}\right) d x d \tau \\
& -\left(\frac{N}{K \beta_{0}}-C-\frac{8 \omega_{1}^{2} \hat{k}(0)}{\alpha L} C_{\delta}\right) \int_{0}^{T} \int_{0}^{L}\left|\hat{k} * \varphi_{x}\right|^{2} d x d \tau \\
& +\frac{8 \omega_{1}^{2} \hat{k}(0)}{\alpha L} \delta \int_{0}^{T} \int_{0}^{L}\left|v_{t}\right|^{2}+\left|v_{x}\right|^{2}+|\varphi|^{2} d x d \tau \\
& +N c \gamma \int_{0}^{T} \int_{0}^{L}\left|v_{t}\right|^{2}+\left|v_{x}\right|^{2}+|\varphi|^{2} d x d \tau \\
& +c \gamma \int_{0}^{T} \int_{0}^{L}\left|v_{t}\right|^{2}+\left|v_{x}\right|^{2}+|\varphi|^{2}+\left|\hat{k} * \varphi_{x}\right|^{2} d x d \tau .
\end{aligned}
$$

Choose $\delta>0$ such that $\frac{8 \omega_{1}^{2} \hat{k}(0)}{\alpha L} \delta<\frac{k_{0}}{4}$. Taking $N$ large enough and $\gamma$ sufficiently small we conclude that

$$
\mathcal{L}(T)-\mathcal{L}(0) \leq-\frac{k_{0}}{2} \int_{0}^{T} \int_{0}^{L}|\varphi|^{2}+\left|v_{x}\right|^{2}+\left|v_{t}\right|^{2} d x d \tau<0
$$

which implies

$$
\mathcal{L}(T) \leq \mathcal{L}(0)
$$

Repeating the above integration from 0 to $n T$ with $n \in \mathbb{N}$ we conclude that

$$
\mathcal{L}(n T) \leq \mathcal{L}(0)
$$

Observe that there exist positive constants $c_{0}$ and $c_{1}$ satisfying

$$
c_{0} e^{-2 \gamma t} E(t ; u, \theta) \leq \mathcal{L}(t) \leq c_{1} e^{-2 \gamma t} E(t ; u, \theta) .
$$


Since any number $t>0$ can be written $t=n T+r$ where $r<T$ and $E(t ; u, \theta)$ is a decreasing function, then from (4.28) and (4.27) it follows that there exists a positive constant $C_{0}$ such that

$$
E(t ; u, \theta) \leq E(n T ; u, \theta) \leq \frac{1}{c_{0}} e^{-2 \gamma n T} \mathcal{L}(n T) \leq C_{0} e^{-2 \gamma n T} E(0 ; u, \theta) .
$$

Since $t \leq 2 n T$, we have

$$
E(t ; u, \theta) \leq C_{0} e^{-\gamma t} E(0 ; u, \theta)
$$

The proof is now complete.

Acknowledgments. The authors express their appreciation to the referee for his invaluable suggestion which improved this paper.

\section{REFERENCES}

[1] B. D. Coleman and M. E. Gurtin, Equipresence and constitutive equations for rigid heat conductors, Z. Angew. Math. Phys. 18, 199-208 (1967)

[2] C. M. Dafermos, An abstract Volterra equation with applications to linear viscoelasticity, Journal of Differential Equations 7, 554-569 (1970)

[3] G. Dassios and F. Zafiropoulos, Equipartition of energy in linearized 3-D viscoelasticity, Quart. Appl. Math. 48, 715-730 (1990)

[4] M. Fabrizio and B. Lazzari, On the existence and asymptotic stability of solutions for linearly viscoelastic solids, Arch. Rational Mech. Anal. 116, 139-152 (1991)

[5] C. Giorgi and M. G. Naso, On the exponential stability of linear non-Fourier thermoviscoelastic bar, qquaderni del Seminario di Brescia 2/97 (1997)

[6] M. E. Gurtin and A. C. Pipkin, A general theory of heat conduction with finite wave speeds, Arch. Rational Mech. Anal. 31, 113-126 (1968)

[7] J. E. Lagnese, Asymptotic energy estimates for Kirchhoff plates subject to weak viscoelastic damping, International Series of Numerical Math. 91, 211-235 (1989)

[8] Z. Liu and S. Zheng, On the exponential stability of linear viscoelasticity and thermoviscoelasticity, Quart. Appl. Math. 54, 21-31 (1996)

[9] J. L. Lions, Quelques méthodes de résolution des problèmes aux limites non linéaires, Dunod, Paris, 1969

[10] J. E. Muñoz Rivera, Asymptotic behaviour of energy in linear thermoviscoelasticity, Computational and Appl. Math. 11, 45-71 (1992)

[11] J. E. Muñoz Rivera, Global smooth solutions for the Cauchy problem in nonlinear viscoelasticity, Differential Integral Equations 7, 257-273 (1994)

[12] J. E. Muñoz Rivera, Asymptotic behavior in linear viscoelasticity, Quart. Appl. Math. 52, 629-648 (1994)

[13] J. E. Muñoz Rivera and E. Cabanillas, Decay rates of solutions of an anisotropic inhomogeneous $n$-dimensional viscoelastic equation with polynomial decaying kernels, Comm. Math. Physics 177, 583-602 (1996)

[14] J. E. Muñoz Rivera, E. Cabanillas, and R. Barreto, Decay rates for viscoelastic plates with memory, Journal of Elasticity 44, 61-87 (1996)

[15] O. J. Staffans, On a nonlinear hyperbolic Volterra Equation, Siam J. Math. Anal. 11, 793-812 (1980) 